\title{
Plasma amino acid profiles suggest changes in one-carbon metabolism in lactating rats fed a diet reflecting that of the poorest socioeconomic group in Scotland
}

\author{
H. Dasgin, S.M. Hay and W.D. Rees \\ Rowett Institute, University of Aberdeen, Foresterhill Aberdeen, AB25 2ZD, Scotland UK
}

Pregnant women in deprived populations in Scotland have a high intake of saturated fat, refined carbohydrates and salt but at the same time low intakes of several micronutrients. Multiple mild deficiencies of micronutrients involved in lipid metabolism such as choline and B vitamins, may exacerbate the adverse effects of excess energy and be part of a mechanism which programs long-term disease risk in the offspring ${ }^{(1)}$. This hypothesis has been tested using the rat as an experimental model. The period of lactation is important for neonatal development and is also the time when the mother experiences a substantial metabolic load. Since nutrient interactions may change amino acid metabolism ${ }^{(1)}$, we have measured concentrations of key metabolites in the plasma of lactating rats.

We have prepared two experimental diets; the first (SIMD) reflecting the median intakes of pregnant women in the tenth decile of the Scottish index of multiple deprivation ${ }^{(2)}$ and compared this with a second diet (SACN) formulated to incorporate the recommendations of the Scientific Advisory Committee on Nutrition i.e. low in saturated fat and sugar, with reduced salt and with micronutrients adjusted to meet the recommended intakes. The SIMD and SACN experimental diets contained $16.4 \%$ protein (w/w) as a mixture of casein and gluten, compared to AIN-93 G reference diet which contained 20\% (w/w) casein. Three groups of rats were maintained on either SIMD, SACN or AIN-93 G diet for a 3-week adaptation period, and for the duration of gestation and lactation. Lactating dams were killed on postnatal day 19 and blood samples were collected by cardiac puncture. Plasma glucose and lipids were measured with a clinical analyser (Kone). Plasma amino acid concentrations were determined by isotope dilution, measured by electron impact GC-MS ${ }^{(3)}$. Data was analysed by one-way ANOVA followed by Fisher's multiple comparison test.

Concentrations of glucose, cholesterol, free fatty acids and triglycerides were not different between SIMD and SACN groups. The concentrations of arginine (SIMD 66.4 \pm 7.2, SACN 107.3- \pm 7.7 , AIN-93 G $122.3 \pm 18.2 \mathrm{nmol} / \mathrm{g}, \mathrm{p}=0.016)$ and glycine (SIMD 139.4 $\pm 10.8, \mathrm{SACN} 209.9 \pm 14.3$, AIN-93 G $191.0 \pm 11.5 \mathrm{nmol} / \mathrm{g}, \mathrm{p}=0.005)$ were reduced in the maternal plasma of the SIMD compared to the SACN and AIN-93 G groups. Concentrations of the remaining amino acids were not different between the SIMD and SACN groups.

Glycine and arginine are key components of methyl metabolism. Glycine ${ }^{(1)}$ is a source of methyl groups required to produce $\mathrm{C}_{1}$ folate derivatives. Arginine is a precursor for creatine synthesis, one of the major reactions consuming methyl groups ${ }^{(3)}$. These changes in plasma glycine and arginine in the SIMD group suggest altered maternal one-carbon metabolism when animals are fed energy dense micronutrient deficient diets during lactation. This may indirectly, alter the supply of key products of maternal methyl metabolism, such as phosphatidylcholine, to the neonate.

1. Rees WD. (2019) Proc Nutr Soc 78, 88-96

2. Haggarty P, Campbell DM, Duthie S, et al. (2009) Br J Nutr 102, 487-97

3. Calder, A. G., et al. (1999). Rapid Commun Mass Spectrom 13, 2080-2083

4. Wu, G., et al. (2018). Journal of Animal Science 96, 5035-5051 